\title{
WATER TREATMENT BY OZONATION: CONTAMINANTS CONCENTRATION ESTIMATION BY DYNAMICAL NEURAL NETWORK
}

\author{
Isaac Chairez, Alexander Poznyak * \\ and Tatyana Poznyak ** \\ *CINVESTAV,IPN. México D.F.E-mail: apoznyak@ctrl.cinvestav.mx. \\ ** ESIQIE, IPN. México, D.F. Email: tpoznyak@hotmail.com
}

\begin{abstract}
A real data set from model mixtures water treatment is considered to be analyzed trough this observer based on dynamical neural networks and sliding mode-like design. The residual sign term is suggested to be used to reduce the output external noise effect in the estimation process and the dynamic neural network is employed to reconstruct the state dynamics of the system under this study. Copyright (C) 2005 IFAC
\end{abstract}

Keywords: Environmental systems, Dynamic neural network, Sliding mode observer, Observer, Ozonation process,

\section{INTRODUCTION}

Ozone has been used intensively in water and waste water treatment, disinfection, bleaching and industrial oxidation processes (Poznyak et al., 2003). The practical applications are typically based on the very high oxidizing power of ozone and its decomposition products (Gordon et al., 1999). Ozonation of water and waste water is typically carried out in semibatch reactors by dispersing gas, which contains dissolved ozone into the liquid phase. Mass transfer between the phases in question can be described using many mathematical models (Bin and Roustand, 2000) and (Poznyak and Vivero, 1998). Ozone absorption in aqueous solutions is accompanied by chemical reactions of different kind and nature. This problem has been widely discussed in literature (Gilbert et al., 1999) and the film theory of mass transfer can be applied for solving the problem. Moreover, it's important to know how the presence of the chemical reaction has a relevant impact on ozone mass transfer process.

\subsection{Precedents}

A lot of efforts have been devoted to analyzing of a certain type of ozone reactions in liquid phases with different organic contaminants (Razumovskii and
Zaikov, 1994), (Poznyak et al., 2003), and (Poznyak et al., 1977). Many modern chemical problems demand the employment of few control approaches, that can provide more detailed understanding of complex chemical phenomena in total. Although the basic concepts on identification theory are quite straight forward, it turns out to be often most time consuming step in the design and implementation of advanced control technologies.

The identification problem for some classes of chemical kinetic models just give a poor example of such situations, which can be abstracted to the problem of a class of nonlinear systems with noncomplete on-line measurable variables (Garcia and D’Atellis, 1995), (Knobloch et al., 1993). Available observations provides by a ozone sensor which supplies the current information in continuous time about the current ozone concentration in the gas phase of the reactor output. The situation which is know, differs in many aspects from standard approaches, commonly used in identification theory: usually mathematical models are assumed to be completely measurable. Among them the assumption that all states are completely accessible is usually accepted. In practice, this assumption doesn't seem to be realistic. That's why the observation process (or state estimation) seems to be very important for effective control design. 
Several approaches dealing with state estimation problem are widely used in practical applications. For instance, there exists the adaptive observation process (Michalska and Mayne, 1995), Lyapunov-like observers approach (Zak and Walcott, 1990), high gain observer (Giccarella et al., 1993), optimizationbased observer (Nicosia and Tornambe, 1989), reduced order nonlinear observers (Krener and Isidori, 1983) and others.

Artificial neural networks have showed good behavior when nonlinear systems (affected by some kind of external noises or perturbations) are trying to be identified. When a mathematical model is incomplete or partially known, the DNN approach (Poznyak et al., 2001) provides an effective instrument to attack a wide spectrum of control problems (such as identification, state estimation, trajectories tracking, etc.). Recently, the design of control functions under heavy uncertainty conditions is one of the main problems of the modern control theory. One of the common approaches is the variable structure control approach (VSC), dealt with the sliding mode control (Utkin, 1992) is widely used due to its insensitivity to external and internal disturbances.

Variable structure control offers significant potential advantages: good transient behavior, global exponential stability, (no modeled) disturbance rejection capability, insensitivity to plant nonlinearity or parameter variations and remarkable stability and performance robustness. In order to exploit these strategies, a suitable estimate of the states should be constructed for use in the control function developed. Despite fruitful research in the variable structure control theory a few authors have considered the application of the main principles of sliding mode control to the problem of observer design. For deterministic system the earliest work in this field has appeared in Utkin (1992), the approach described in it is similar to that proposed by Slotine (1984).

The design and control of ozonation reactions have been the challenging tasks mostly, because of the inadequacy of on-line sensors with fast sampling rate and small time delay and the complex nonlinear interactive behavior of ozonation reaction. For these reasons, it can be assumed that control techniques will be used in order to avoid these limitations. On the other hand, there are not adequate mathematical models of ozonation reaction when it is driven at basic $\mathrm{pH}(\geq 7.0)$, because here two different mechanisms are present: direct attack by molecular ozone to the organic compound and indirect reaction with $\mathrm{OH}$ radicals.

Then the problem to solve is as follows: based on online experimental data (ozone gas concentration on the reactor output), to construct the immeasurable dynamics (using a DNN-observer) of contaminates decomposition at different $\mathrm{pH}(2.0,7.0$ and 12.0)
(Poznyak et al., 2001) and compare with experimental data acquired with classical techniques for model mixtures.

The paper is outlined as follows. In section 3, the class of nonlinear systems, which is going to be treated along this paper, is introduced. The basic assumptions concerning the behavior of the considered system and external disturbances are presented too. The main result in this work is developed in section 2 also, it gives an upper bound for the averaged estimation error as a function of an uncertainty level in the output measures (given by the features of the ozone sensor) as well as some a priory known characteristics of the considered model. Section 3, where the numerical example dealing with ozonation reaction dynamics is considered, concludes this study.

\subsection{Ozonation Model Description}

Ozone is capable to oxidize a wide variety of organic materials in aqueous solution. The oxidation process by ozone involves the phenomenon of mass transfer with simultaneous chemical reaction. Building on results of (Lisitsin et al., 1976), (Poznyak and Vivero, 1998), dealing ozonation with ith-component model mixture, we can write the following system of $N+2$ differential equations:

$$
\begin{aligned}
& \frac{d}{d t} c_{t}^{g a s}=\frac{\omega^{g a s}}{v^{g a s}}\left[c_{0}^{\text {gas }}-c_{t}^{\text {gas }}\right]-\frac{1}{v^{\text {gas }}} K_{\text {sat }} \Delta Q_{t} \\
& \frac{d}{d t} Q_{t}=K_{s a t} \Delta Q_{t}-\sum k_{i} c_{t}^{i} Q_{t} \quad \Delta Q_{t}:=Q_{\max }-Q_{t} \\
& \frac{d}{d t} c_{t}^{i}=-\frac{k_{i} c_{t}^{i} Q_{t}}{v^{\text {liq }}}(i=1,2, \ldots, N)
\end{aligned}
$$

Where $c_{t}^{\text {gas }}[$ mole/L] is the ozone concentration in the gas phase (this is ozone which doesn't react with organic compounds dissolved in the solvent), $Q_{t}$ [mole] is the ozone dissolved in liquid phase, $c_{t}^{i}$ $[m o l e / L]$ is the organic compound concentration at time $t$ for the ith-component in the model mixture and $N$ is the number of contaminants in the reactor. The parameters, involved in the ozonation model description, have the following physical sense: $v^{\text {gas }}$ $[L]$ is the volume of gas phase which is assumed to be constant, $\omega^{\text {gas }}[L / s]$ is the input gas flow, $K_{\text {sat }}[1 / s]$ is the saturation constant, $Q_{\max }[$ mole $]$ is the maximum of ozone being in the saturated liquid phase under the given conditions, $k_{i}\left[L /\left(s^{*}\right.\right.$ mole $\left.)\right]$ is the ith rate constant of ozone with the organic compound, $v^{\text {liq }}[L]$ is the liquid phase volume. Notice that for this work, all components can not be available to measure online, the only one measurable state of this process is $c_{t}^{\text {gas }}$. 


\section{OBSERVER BASED DYNAMIC NEURAL NETWORK}

To develop an estimation technique based on neural networks and sliding mode technique, the following facts should be taken into account:

a. The ozonation reaction system is stable.

b. The control functions for ozonation are bounded.

Both assumptions seem to be natural for the ozonation process under consideration. Take into account the following class of nonlinear systems including the ozonation mathematical model given by (2):

$$
\begin{aligned}
& \dot{x}(t)=\dot{x}_{t}=f\left(x_{t}, u_{t}, t\right)+\xi_{1, t} \\
& y(t)=y_{t}=C x_{t}+\xi_{2, t}
\end{aligned}
$$

where $x_{t} \in \mathfrak{R}^{n}$ is the state vector of the system, $u_{t} \in \mathfrak{R}^{m}$ is a given control action, $y_{t} \in \mathfrak{R}^{p}$ is the output signal which is assumed to be measurable at each time, $C: \mathfrak{R}^{p \times n}$ is a priory known output matrix $f\left(x_{t}, u_{t}, t\right): \mathfrak{R}^{n} \times \mathfrak{R}^{m} \times \mathfrak{R} \rightarrow \mathfrak{R}^{n}$ is an unknown vector nonlinear function, describing the system behavior and satisfying some conditions about existence and uniqueness for the solution of (2). The vectors $\xi_{1, t}$ and $\xi_{2, t}$ represent external partially known bounded perturbations $\left\|\xi_{i, t}\right\|_{\Lambda_{\xi_{i}}} \leq \Upsilon_{i} \prec \infty$ for the state and output dynamics, respectively. The suggested sliding mode neural-observer has the following structure (Poznyak, 2004)

$$
\begin{gathered}
\frac{d \hat{x}_{t}}{d t}=A \hat{x}_{t}+W_{1} \sigma\left(V_{1} \hat{x}_{t}\right)+W_{2} \varphi\left(V_{2} \hat{x}_{t}\right) \gamma\left(u_{t}\right)+ \\
K_{1} \operatorname{sign}\left(y_{t}-\hat{y}_{t}\right) ; \quad \hat{y}_{t}=C \hat{x}_{t}
\end{gathered}
$$

Here the vector $\hat{x}_{t} \in \mathfrak{R}^{n}$ is the state of the neural network, $u_{t} \in \mathfrak{R}^{m}$ is its input. The matrix $A \in \mathfrak{R}^{n \times n}$ is a stable fixed matrix which will be specified below. The matrices $W_{1, t} \in \mathfrak{R}^{n \times s_{1}}$ and $W_{2, t} \in \mathfrak{R}^{n \times t_{1}}$ are the weights of the output layers. $V_{1, t} \in \mathfrak{R}^{s_{2} \times n}$ and $V_{2, t} \in \mathfrak{R}^{t_{2} \times n}$ are the weights of the internal hidden layer. $K_{1}$ is the gain matrix for the sign term. The activation functions for each neuron in the output layer $\sigma(\bullet)$ and in the hidden layer $\varphi(\cdot)$ are selected as sigmoid functions:

The initial conditions are fixed by the ozonation reaction features, based on the available data about the experiments. Now, at this point we suppose that the neuro-observer fulfills the following:
Assumption A1: There exists a stable matrix $A$ and a positive parameter $\delta$ such that the following matrix Riccati equation has a positive solution $\mathrm{P}=\mathrm{P}^{\mathrm{T}}>0$ :

$$
\begin{gathered}
P A+A^{T} P+P R P+Q=0 \\
R:=2 \bar{W}_{1}+2 \bar{W}_{2}+\Lambda_{9}^{-1}+\Lambda_{10}^{-1} \\
Q:=\delta\left(\Lambda_{4}^{-1}+\Lambda_{6}^{-1}+\Lambda_{15}^{-1}+\Lambda_{17}^{-1}\right)+\Lambda_{\sigma} \\
+\bar{u}^{2} \Lambda_{\varphi}+k \delta I_{n \times n}+P_{1}+k \delta \Xi I_{n \times n}+Q_{0} \\
+C^{T}\left(\xi_{2}^{-1} \Lambda_{18}^{-1}\left(\xi_{2}^{-1}\right)^{T}-\Lambda_{\xi_{2}}\right) C
\end{gathered}
$$

The weights matrices of the considered DNNobserver are adjusted according to the following differential equations (Learning laws) for external layer:

$$
\begin{gathered}
\dot{W}_{1}=-k_{1} P N_{\delta} C^{T} e_{t}\left[\sigma\left(V_{1, t} \hat{x}_{t}\right)^{T}-\frac{1}{2} \hat{x}_{t} V_{1, t} D_{\sigma}^{T}\right] \\
-\frac{k_{1}}{2} P N_{\delta}\left[\delta C^{T} \Lambda_{3} C+\Lambda_{4}\right] N_{\delta} P \tilde{W}_{1, t} \sigma\left(V_{1, t} \hat{x}_{t}\right) \sigma\left(V_{1, t} \hat{x}_{t}\right)^{T} \\
\dot{W}_{2}=-k_{2} P N_{\delta} C^{T} e_{t}\left[\gamma\left(u_{t}\right)^{T} \varphi\left(V_{2, t} \hat{x}_{t}\right)^{T}-\frac{1}{2} \hat{x}_{t} V_{2, t} D_{\varphi}^{T}\right] \\
-\frac{k_{2}}{2} P N_{\delta}\left[\Lambda_{6}+\delta C^{T} \Lambda_{5} C\right] N_{\delta} P \tilde{W}_{1, t} \varphi\left(V_{1, t} \hat{x}_{t}\right) \gamma\left(u_{t}\right) \gamma\left(u_{t}\right)^{T} \varphi\left(V_{1, t} \hat{x}_{t}\right)^{T} \\
\dot{V}_{1}=-\frac{k_{3}}{2} D_{\sigma}^{T}\left(W_{1}^{*}\right)^{T} P N_{\delta}\left(\delta \Lambda_{15}+C^{T} \Lambda_{16} C\right) N_{\delta} P W_{1}^{*} D_{\sigma} \tilde{V}_{1, t} \hat{x}_{t} \hat{x}_{t}^{T} \\
-\frac{k_{3}}{2} D_{\sigma}^{T} W_{1, t}^{T} P N_{\delta} C^{T} e_{t} \hat{x}_{t}^{T}-\frac{k_{3}}{2} 1_{1} \Lambda_{7} \tilde{V}_{1, t} \hat{x}_{t} \hat{x}_{t}^{T} \\
\dot{V}_{2}=-\frac{k_{4}}{2} D_{\varphi}^{T}\left(W_{2}^{*}\right)^{T} P N_{\delta}\left(\delta \Lambda_{17}+C^{T} \Lambda_{18} C\right) N_{\delta} P W_{2}^{*} D_{\varphi} \tilde{V}_{2, t} \hat{x}_{t} \hat{x}_{t}^{T} \\
-\frac{k_{4}}{2} D_{\varphi}^{T} W_{2, t}^{T} P N_{\delta} C^{T} e_{t} \hat{x}_{t}^{T}-\frac{k_{4}}{2} l_{2} \Lambda_{8} \tilde{V}_{2, t} \hat{x}_{t} \hat{x}_{t}^{T}
\end{gathered}
$$

Where:

$$
\tilde{W}_{i, t}:=W_{i, t}-W_{i, t}^{*}, \tilde{V}_{i, t}:=V_{i, t}-V_{i, t}^{*} \quad(i=1,2) .
$$

Based of the neuro-observer structure and the assumptions given above, the next fact can be formulated.

Theorem 1 If the nonlinear system given by (2) satisfies the assumptions given below, and the neural observer given by (3) satisfies the assumption (A1) too, using the learning laws (5), then the following upper bound for the averaged estimation error holds:

$$
\begin{gathered}
\varlimsup_{T \rightarrow \infty} \frac{1}{T} \int_{t=0}^{T}\left\|\Delta_{t}\right\|_{P} d t \leq\left(\frac{\rho_{Q}}{\left(k \sqrt{\alpha_{P}}+\sqrt{k^{2} \alpha_{P}+\alpha_{Q} \rho_{Q}}\right)}\right) \\
\rho_{Q}:=\eta_{1}+\Upsilon_{1}+7 \Upsilon_{2}+4 k \sqrt{N}\left\|\xi_{2, t}\right\|+k \Xi \\
\alpha_{P}:=\lambda_{\min }\left(P^{-1 / 2}\left[C^{T} C+\delta I\right] P^{-1 / 2}\right)>0 \\
\alpha_{Q}:=\lambda_{\min }\left(P^{-1 / 2} Q_{0} P^{-1 / 2}\right)>0
\end{gathered}
$$


Comment If the external disturbances for state and output dynamics are absent, then we guarantee that the estimates converge asymptotically to their real states.

\section{ILLUSTRATIVE EXAMPLES}

3.1 Example 1 (with data generated by the ozonation mathematical model)

Using the ozonation reaction mathematical description (1) to generate the simulated data set of ozonation variables, its possible to apply the neural observer (Fig. 1) given in (3) to reconstruct the organics decomposition dynamics based only in the measurable data $c_{t}^{\text {gas }}$.

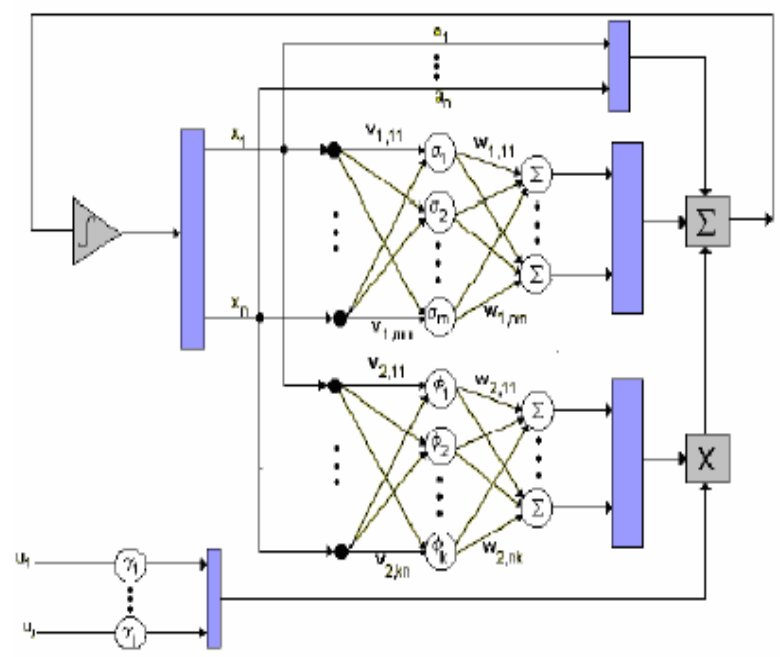

Fig. 1 Neural Observer structure.

An illustrative case is studied now: the model mixture $\left(k_{1}=1.3 \times 10^{3}, k_{2}=2.5 \times 10^{3} \mathrm{~L} / \mathrm{mol}^{*} \mathrm{~s}\right)$ of two organics (phenol and 2-chlorophenol) is dissolved in liquid phase, and their decomposition dynamics have been calculated (Figs. 3 and 4) using the observer based on the measurable online data ( $\left.c_{t}^{\text {gas }}\right)$ (Fig. 2). The time evolution given by this example shows how the neural network $\left(a_{11}=-3.6, a_{12}=0\right.$, $a_{21}=3.2, a_{22}=-9.9, \quad W_{i j}^{1,2} \in[0.8,3.2] \quad$ and $\left.V_{i j}^{1,2} \in[0.3,0.8]\right)$ reaches the dynamics generated by the ozonation mathematical model.

This process is complete in two stages, the first one is known as learning phase when the neural network has its adaptation behavior $(\mathrm{t} \leq 20 \mathrm{~min}$.). The second age is given by an exact correspondence between the neural network and the ozonation dynamics. Obviously the $\mathrm{Ct}$ gas time evolution is identified based exactly on available data, but in the contaminants case, they are reconstructing based on inexactly data but on neural network with observer structure.

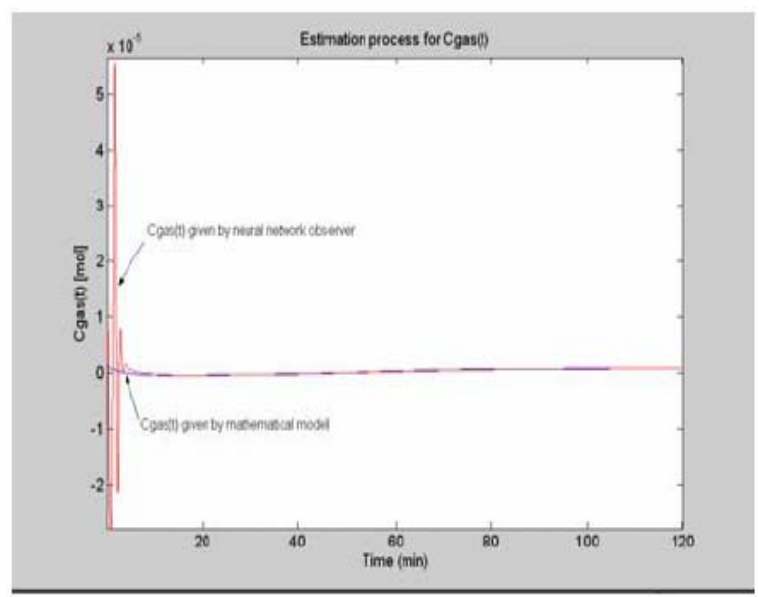

Fig. $2 c_{t}^{\text {gas }}$ estimation given by the neural observer suggested using data given by the mathematical model.

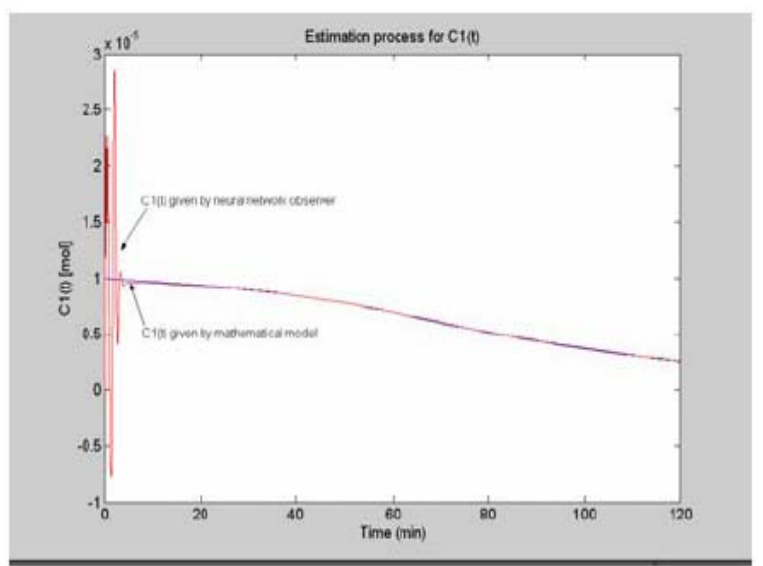

Fig. $3 c_{t}^{1}$ estimation given by the neural observer suggested using data given by the mathematical model.

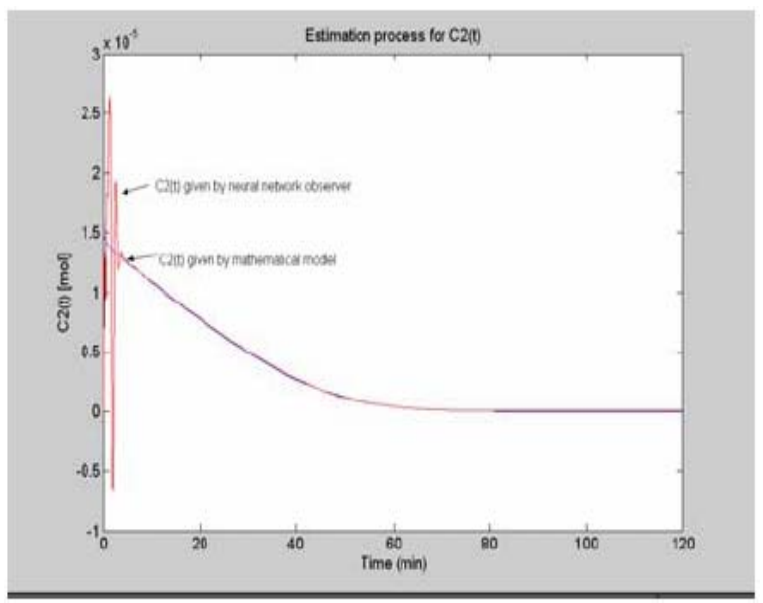

Fig. $4 c_{t}^{2}$ estimation given by the neural observer suggested using data given by the mathematical model.

The performance index related with the error estimation process has a fast decrement (Fig. 5). This portrait implies a real converge between real states and delivered by DNN. 
Analytical methods.

Two different model mixtures were reacted with ozone during 50 minutes. The data obtained with a ozone sensor were analyzed in a Personal Computer. Both data series were treated with the neural observer using the contaminants concentration given by a high performance liquid chromatography (HPLC) without the use of any mathematical model.

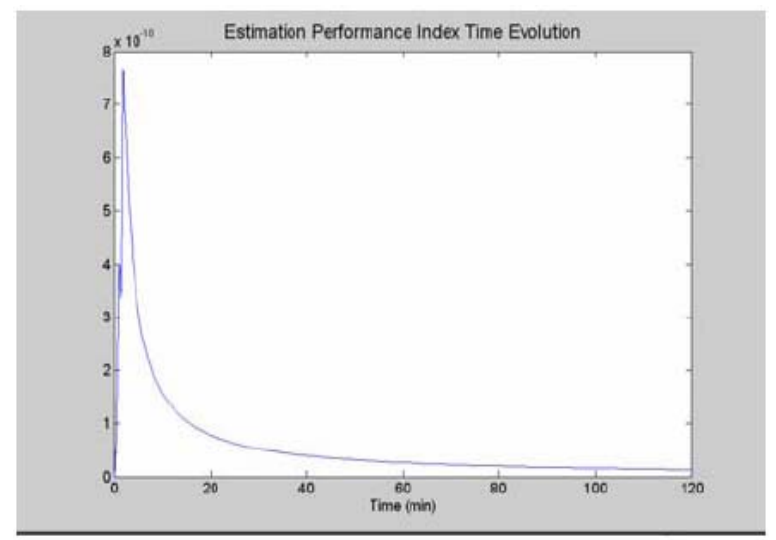

Fig. 5 Error function time evolution between the model dynamics and the DNN approach for two contaminants.

\section{Material and methods}

The ozonation of phenols solution (phenol, 4chlorophenol and 2,4 dichlorophenol) with the initial concentration $(50 \mathrm{mg} / \mathrm{L})$, was carried out in a semibatch reactor $(0.5 \mathrm{~L})$ using an ozone generator "AZCO" with the initial ozone concentration $25 \mathrm{mg} / \mathrm{L}$ and the gas flow $0.5 \mathrm{~L} / \mathrm{min}$. The measurements of ozone in gas phase at the output of the reactor was done with an ozone sensor model BMT 930, connected to a PC in order to construct the ozonation reaction experimental curve in Matlab. Different series of ozonation at neutral initial $\mathrm{pH}$ (7.0) and basic $\mathrm{pH}$ (12) were studied. In figure (Fig. 6) the schematic diagram of ozonation apparatus is shown.

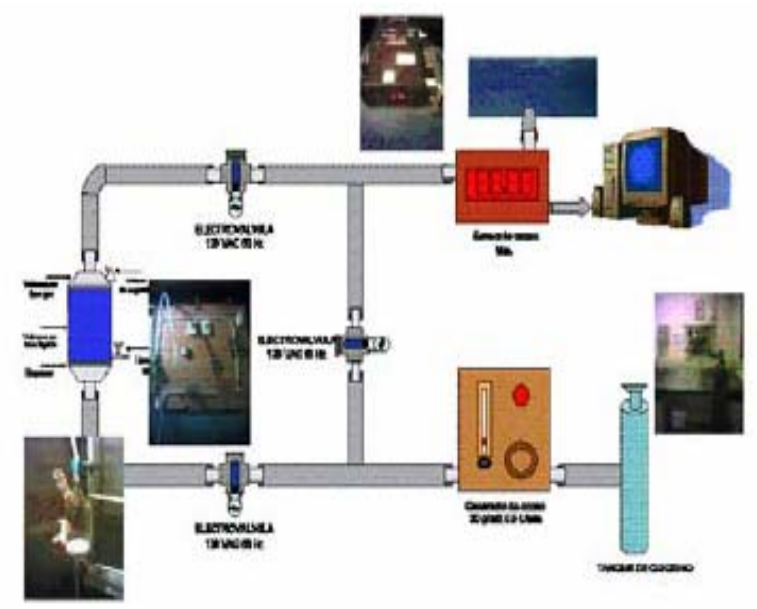

Fig. 6 Ozone real system using in the experiments at different $\mathrm{pH}$ 's.
To verify the quality of the obtained estimates, the aqueous samples were analyzed by HPLC. The HPLC analysis was made a liquid chromatography PerkinElmer series 200 coupled with a automatic UV/VIS detector with a chromatography column Nova Pack C-18, 250*4.6 mm with a mobile phase of water: acetonitrile: phosphoric acid mixture (50:49:1) with the flow rate of $0.8 \mathrm{~mL} / \mathrm{min}$. The corresponding organic compound concentration was measured in different reaction times, in order to use it to compare with the decomposition dynamic generated by the observer. Applying the suggested DNN-observer containing the additional sign correction term, we obtain (see Fig. 8) a faster convergence of the state estimation error which turned out to be better than the one obtained in (Poznyak et al., 2001). Even more, when some external disturbances (as ozone concentration measurement noises) (Fig. 7) are presented, this estimator converge into a bounded zone around the real state time dynamics, lesser than those given in (Poznyak et al., 2001) for example.

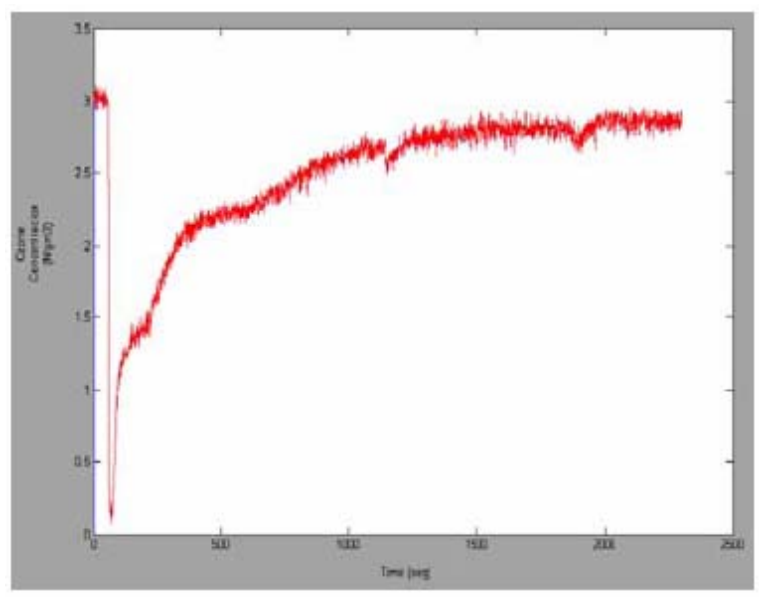

Fig. 7 Comparison of the experimental $c_{t}^{\text {gas }}(+)$ and DNN behavior of the two organic compounds decomposition reaction (phenol (1), 2chlorophenol (2)) at $\mathrm{pH}=7.0$.

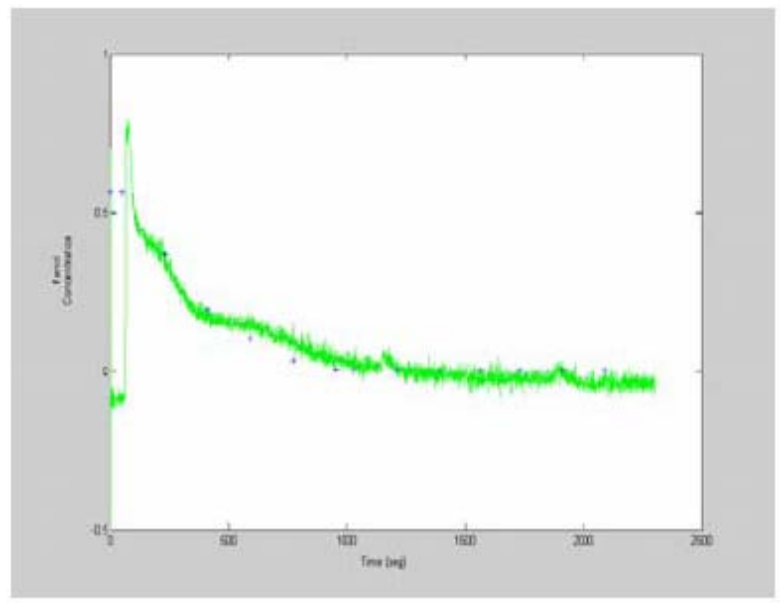

Fig. 8 Comparison of the experimental $c_{t}^{1}(+)$ and DNN behavior of the organic compounds decomposition (phenol) at $\mathrm{pH}=7.0$. 
The measured gas phase ozone concentration was reached so fast, meanwhile the continuous portrait for organic compound concentration coincides with the data obtained in experiments. As in the mathematical model case, the performance index is calculated as the average integral for error signal for two different observers (Fig. 9): linear term (solid) and sign term (dashed).

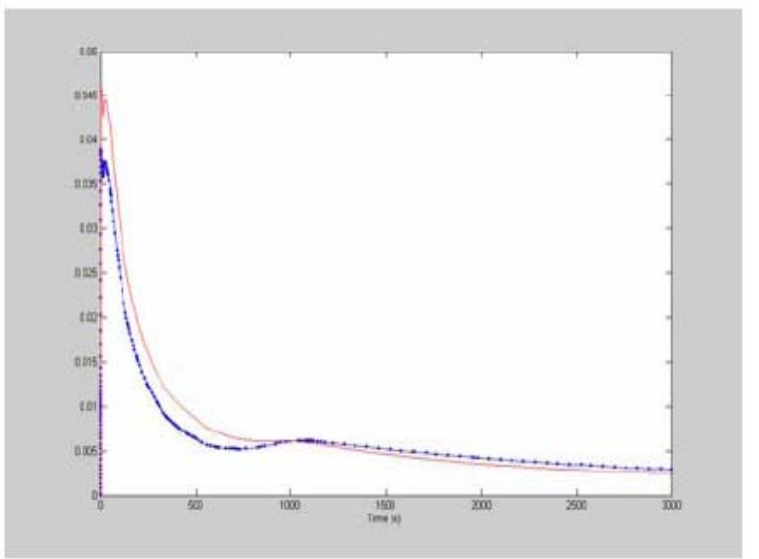

Fig. 9 Error function time evolution between the model dynamics and the DNN approach for two contaminants and two different observers: linear error correction term (solid) and sign error correction term (dashed).

\section{CONCLUSIONS}

The treated approach is shown to be able to guarantee a good state estimation if the suggested DNNobserver with sign correction term is applied. So, the sign terms provoke more estimation quality with respect to a standard observer with a linear correction term. On the other hand, the theoretical analysis shows how we may improve (to make lesser) the upper bound (6) for the estimation error varying the involved free parameters.

\section{REFERENCES}

Bin, A.K. and M. Roustand (2000). Mass transferring ozone reactors. In: International Specialized Symposium: Fundamental and Engineering Concepts of Ozone Rector Design. IOA/EA3G. France. pp. 97-131.

Garcia, R.A. and C.E. D'Atellis (1995). Trajectory tracking in nonlinear systems via nonlinear reduced-order observers. International Journal of Control 62, 685-715.

Giccarella, G., M.D. Mora and G. Germani (1993). A Luenberger-like observer for nonlinear systems. International Journal of Control. 57, 537-556.

Gilbert, G., N. Attila and Istvan F. (1999). Evaluation of kinetics models for ozone decomposition: limitations and perspectives. In: 14 th ozone world congress. Int. Ozone Assoc. Pan American Group. Michigan USA.

Gordon, G., A. Nemes and F. Istvan (1999). Evaluation of kinetics models for ozone decomposition: limitations and perspectives. In: 14 th Ozone World Congress. Michigan, USA.

Knobloch, H. W., A. Isidori and D. FLocherzi (1993). Topics in Control Theory. Birkhauser Verlag. Basel-Boston-Berlin.

Krener, A.J. and A. Isidori (1983). Linealization by output injection and nonlinear observers. Systems and Control Letters 3, 47-52.

Lisitsin, Poznyak T.I. and S.D. Razumovskii (1976). Methematical modeling of the reaction of ozone with some organic compoundsin a continuos operation bubbling reactor. Kinet. Kalac. 17, 1049-1056.

Michalska, H. and D.Q. Mayne (1995). Moving horizons observers and observer-based contro. IEEE Transactions on Automatic Control 40, 995-1006.

Nicosia, S. and A. Tornambe (1989). High gain observer in the state and parameter estimations of robots having elastic joins. Systems and Control Letters 13, 331-337.

Poznyak, A.S. (2004). Deterministic Output Noise Effects in Sliding Mode Observation (Ch. 3). In Variable Structure Systems: From Principles to Implementation. Sabanovic, a. and Fridman, 1. and Spurgeon, (eds). ed. Applied Mathematics. London.

Poznyak, A.S., E.N. Sanchez and W. Yu (2001). Dynamic Neural Networks for Nonlinear Control: Identification, State Estimation and Trajectory Tracking. World Scientific. New Jersey, London, Singapore, Hong Kong.

Poznyak, T., A. Manzo and J.L. Mayorga (2003). Elimination of chlorinated unsaturated hydrocarbons from water ozonation: simulation and experimental comparision. Revista de la Sociedad Química de México 47, 58-65.

Poznyak, T.I. and J.L. Vivero (1998). Simulation and optimization of phenol and chlorophenols elimination from wastewater. In: Proceedings of the IOA/Pag. IOA/Pag. Vancouver, Canada. pp. $615-628$.

Poznyak, T.I., D.M. Lisitsyn and D.D. Nokinov (1977). Selective detector for unsturated substances in liquid chromatography, mathematical design of reaction cell. Journal of Analitycal Chemical 11(32), 2218-2226.

Razumovskii, S.D. and G.E. Zaikov (1994). Ozone and its reactions with organic compounds. Elsevier.

Slotine, J.J.E. (1984). Sliding controller design for nonlinear systems. International Journal of Control 40, 421 - 434 .

Utkin, V.I. (1992). Sliding Modes in Control Optimization. Springer-Verlag. Berlin.

Zak, H. and B. L. Walcott (1990). State observation of nonlinear control systems via the method of Lyapunov. pp. 333-350. A.S.I.. Stevenage UK. 